\title{
El fin de la ventaja del jugador único: un experimento*
}

\author{
Laura Ferrari Bravo \\ Universidad de Roma \\ Luigi Luini \\ Universidad de Siena
}

\section{Resumen}

Presentamos un experimento sobre fijación de precios en un duopolio con costes de producción simétricos y cuotas iniciales de mercado asimétricas. Las empresas compiten durante 15 rondas enfrentándose a una demanda simulada con inercia, teniendo la posibilidad de asumir un coste de marketing/publicidad para evitar los efectos de la inercia. Demostramos que los sujetos bien alcanzan un equilibrio de Stackelberg y ponen precios diferentes, bien ponen precios parecidos y llegan a la solución de Cournot con elementos de cooperación, o bien la empresa entrante supera a la ya existente (leapfrogging). Proponemos dos aplicaciones potenciales del experimento: (1) competencia posterior a una patente y (2) liberalización de un monopolio natural.

Clasificación JEL: D12, L13, L43.

Palabras clave: liberalización de un monopolio, barreras a la entrada, política de regulación y competencia.

\section{Abstract}

We present an experiment on a price-setting duopoly with symmetric production costs and asymmetric initial market shares. Firms compete for fifteen rounds facing a simulated market demand inertia, with the possibility of incurring into additional fixed marketing/advertising costs in order to offset the inertia effect. We show that subjects either reach a Stackelberg equilibrium and keep pricing differently, either price equally so as to reach a Cournot solution with elements of co-operation, or else the entrant overtakes the incumbent firm (leapfrogging). Finally, we discuss two potential economic applications for the experiment: (1) post-patent competition and (2) liberalization of a former natural monopoly.

JEL classification: D12, L13, L43.

Keywords: monopoly expiration and barriers to entry, regulation and competition policy.

\section{Introducción}

La mayoría de los modelos de entrada suponen que el entrante no toma sus decisiones de manera explícita y que, por lo tanto, deriva sus opciones a partir de una regla ad hoc. Como consecuencia, una de las debilidades más notables de los modelos de entrada es la falta de cualquier referencia explícita a la conducta optimizadora del entrante.

\footnotetext{
* Traducción a cargo de José Manuel Puerta.
} 
Consideremos una versión simplificada del Postulado de Sylos ${ }^{2}$, representado por el proceso líder-seguidor de Stackelberg, en la cual la empresa establecida, al mover primero, establece una condición (generalmente, una inversión en capital fijo) que se transmite luego al entrante. Esta condición es la variable estratégica en la que se basa la ventaja del líder como jugador que decide primero (first mover). Si esa condición es una variable exógena como, por ejemplo, una planta con escala mínima, la empresa establecida puede únicamente decidir si invertir a un coste y capacidad dados, y tanto la entrada bloqueada como la acomodada son alcanzables bajo condiciones conocidas como fijación de precios límite estática. Este tipo de asuntos se representa dentro de un esquema de juego simultáneo.

La versión dinámica de la fijación de precios límite difiere del caso estático en que la empresa establecida, además de su decisión de invertir, determina el tamaño, imperfectamente reversible, de su inversión (Friedman, 1979; Spence, 1979; Dixit, 1980). En este sentido, la inversión se convierte en una variable endógena que se une en la determinación de la estrategia (fijación de precios límite dinámica). El resto de las características del modelo líder-seguidor de Stackelberg se mantienen. Es decir, los equilibrios factibles dependen únicamente de las estrategias de la empresa establecida, mientras que el entrante no tiene ninguna estrategia.

Este comportamiento estratégico por parte de la empresa establecida ha sido llamado «fat-cat effect» (efecto del gato gordo) (Fudenberg y Tirole, 1984). De acuerdo con estos autores, el «efecto del gato gordo» no siempre representa la mejor estrategia para la empresa establecida en el mercado, especialmente cuando la publicidad e I+D se consideran como variables competitivas.

Fue un modelo específico de competencia en publicidad (Schmalensee, 1983) el que inspiró el trabajo de Fudenberg y Tirole. En ese modelo, la empresa establecida postpone la entrada de su competidor al elegir no invertir en publicidad (es decir, una empresa establecida hostil envía la señal de que recortará sus precios en caso de entrada). En caso contrario, si la empresa establecida decide gastar en publicidad, el entrante recibe la señal de que no habrá un comportamiento drástico de precios (la firma establecida se comportará como un gato gordo del tipo blando). En consecuencia, nos enfrentamos a un juego en dos etapas y aparece, para la empresa establecida, el problema de crearse una reputación, es decir, no invertir en publicidad constituye una amenaza creíble de una política de precios dura/agresiva.

Fudenberg y Tirole reconocen que incluso esta última extensión del modelo básico «no se generaliza al caso en el cual ambas empresas deciden estratégicamente». Estos autores discuten sobre el tipo de equilibrio que es general en un juego de señalización de dos dimensiones (precio y publicidad) cuando ambos instrumentos son utilizados por la empresa establecida. Kreps y Wilson (1982) demuestran que, con

\footnotetext{
${ }^{2}$ El término «Postulado de Sylos» fue establecido por Modigliani para indicar la hipótesis subyacente en el trabajo de SYLOS LABINI (1962) sobre los oligopolios homogéneos: «los entrantes potenciales esperan que las empresas establecidas adopten la política más desfavorable hacia ellos». Ver MODIGLIANI (1958), p. 217.
} 
la introducción de «un poco de información imperfecta» tanto para el entrante como para la empresa establecida en el mercado, el papel jugado por la reputación se vuelve importante en juegos repetidos un número finito de veces, puesto que se hace posible evitar una evaluación ad hoc del comportamiento del entrante ${ }^{3}$.

En general, todos estos modelos atribuyen al consumidor un papel en relación a la política publicitaria representado por acciones de buena voluntad, según Dorfman y Steiner (1954) y Nerlove y Arrow (1962). La inversión en publicidad es similar a la inversión en capital fijo, en el sentido de que se deprecia con el tiempo sin ninguna relación directa con la política de precios. Así, al dividir el juego en dos etapas, estos modelos permiten distinguir el efecto de la publicidad (primera etapa) del efecto de la política de precios (segunda etapa).

En un juego de más de dos etapas se puede introducir en el modelo una dependencia temporal estructural, de acuerdo a la sugerencia de Friedman de considerar una «función de demanda en la cual las ventas o los precios pasados afectan la demanda del periodo actual» (Friedman 1983, p. 186). Esto se hace para modelos de oligopolio con persistencia de hábitos en los consumidores. Los resultados muestran la existencia de competencia oligopolística sin nueva entrada. Hoggart (1959), Selten (1965) y Fisher (1988) presentan modelos experimentales donde la persistencia de hábitos se considera explícitamente por medio del concepto de demanda con inercia. Keser $(1992,1993,2000)$ realiza experimentos en esta línea.

La dependencia temporal estructural ha sido también investigada desde una perspectiva empírica. Mueller (1997), por ejemplo, investiga las ventajas generadas desde el lado de la demanda y la oferta. Entre las primeras, este autor encuentra que la inercia se debe a cuatro causas diferentes: (i) costes de instalación y cambio; (ii) externalidades de red; (iii) incertidumbre sobre la calidad y (iv) formación de hábitos. Del lado de la oferta, la eficiencia se sostiene a través de (i) los costes de instalación y cambio para los compradores (políticas activas a fin de crearlos o mantenerlos); (ii) externalidades de red (políticas activas a fin de crearlas o mantenerlas); (iii) economías de escala (en un sentido dinámico), y (iv) reducciones de costes debido al aprendizaje en el puesto de trabajo (learning-by-doing).

Lieberman y Montgomery (1988) distinguen entre las ventajas y las desventajas del jugador que mueve primero. Clasifican como ventajas: (i) liderazgo tecnológico debido a la experiencia en el aprendizaje (diversidad entre los propietarios, lo cual incrementa las ventajas, y spillovers horizontales, los cuales reducen las ventajas), patentes y secretos comerciales así como ventajas organizacionales (nuevas economías de escala tales como la publicidad); (ii) acción preventiva sobre activos tales como el acceso limitado a un input estratégico de los competidores, la ubicación, las características del producto y la planta y el equipo. Las desventajas se deben a: (i) comportamientos oportunistas (free riding) sobre las nuevas inversiones; (ii) la reso-

\footnotetext{
${ }^{3}$ En esta misma línea, véanse también las contribuciones sobre políticas predatorias de precios de ROSENTHAL (1981) y MILGROM y ROBERTS (1982).
} 
lución de las incertidumbres tecnológicas y de mercado; (iii) discontinuidad tecnológica e inercia por parte de la empresa establecida.

La literatura no distingue claramente entre mercados monopolistas y oligopolistas: la(s) empresa(s) superviviente(s) es más antigua que la(s) que no sobrevive(n) y, en los casos en los que hay más de una empresa establecida, todavía no se sabe qué tipo de competencia/cooperación surge entre ellas. Además, en este enfoque existe una dificultad para testar el comportamiento estratégico debido a la escasa confianza que dan los datos sobre beneficios en comparación con las cuotas de mercado.

FIGURA 1

FORMA EXTENSIVA DEL JUEGO

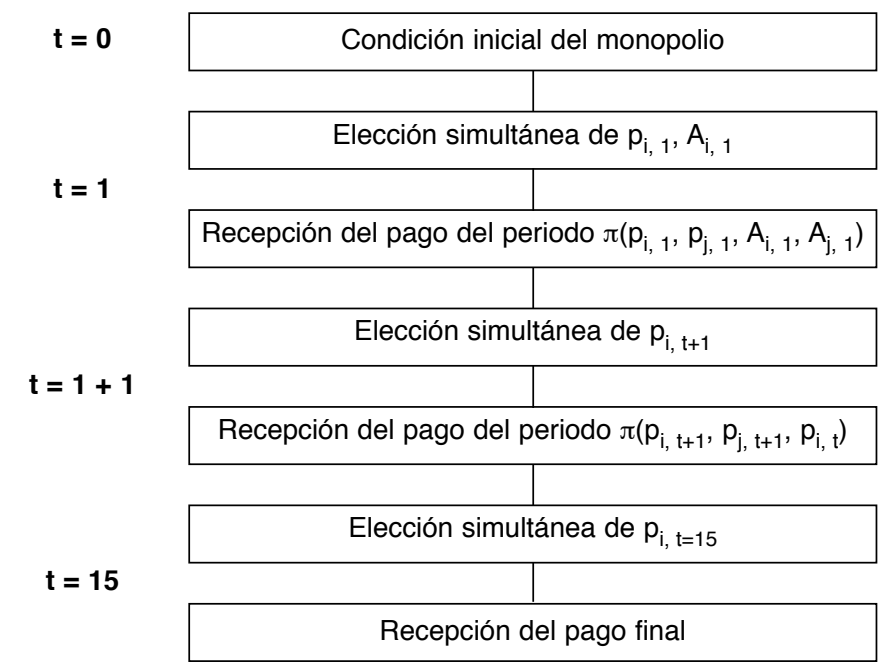

El trabajo que aquí presentamos conecta la literatura sobre políticas dinámicas de precios y publicidad con entrada, con la literatura sobre demanda con inercia. Obtenemos un modelo con entrada con dos variables de señalización (publicidad $\left(\mathrm{A}_{\mathrm{i}}\right)$ y precio $\left(\mathrm{P}_{\mathrm{i}}\right)$ en la Figura 1), bajo la condición de inercia de demanda en un contexto de un juego de múltiples etapas en el que el único que mueve (en la etapa 0) se enfrenta a un nuevo entrante desde la etapa 1 a la 15. La forma extensiva del juego está representada en la Figura 1. Ese modelo se utiliza para investigar experimentalmente el comportamiento de precios de los competidores tras la ruptura de la situación de monopolio.

El artículo está estructurado de la siguiente manera. En la sección 2 describimos la forma extensiva del juego. La sección 3 considera las limitaciones de cálculo impuestas, en cuanto a la solución del juego, tanto por el enfoque evolutivo como por el de teoría de juegos. El marco del experimento se presenta en la sección 4. Finalmente, la sección 5 presenta los resultados experimentales y la sección 6 las conclusiones. 


\section{El modelo}

El juego comienza con una historia del pasado que asigna todo el mercado a una empresa. Llamamos a esta empresa la empresa «Establecida» (Empresa I de aquí en adelante, por su inicial en inglés, Incumbent) mientras que la nueva empresa que entra al mercado se denomina «Entrante» al mercado (firma $E$ de aquí en adelante). El juego dura 15 rondas y se ejecuta bajo dos tipos de demanda, con diferente elasticidad. Llamamos a estos dos tipos de demanda Regulación C y Regulación D. Bajo la Regulación $\mathrm{C}$ los agentes reaccionan rápidamente a las variaciones de los precios, de manera que la elasticidad de la demanda es alta. La Regulación D identifica una demanda más inelástica (Tabla 1).

TABLA 1

FUNCIONES DE DEMANDA DE MERCADO $(I=I, E)$

\begin{tabular}{|c|c|}
\hline Elasticidad alta & Elasticidad baja \\
\hline Demanda de mercado (directa) & Demanda de mercado (directa) \\
$Q_{i}=150-\frac{5}{4} P_{i}$ & $Q_{i}=120-\frac{4}{5} P_{i}$ \\
\hline Demanda de mercado (inversa) & Demanda de mercado (inversa) \\
$P_{i}=120-\frac{4}{5} Q_{i}$ & $P_{i}=150-\frac{5}{4} Q_{i}$ \\
\hline Coste unitario constante & Coste unitario constante \\
$c_{i}=20$ & $c_{i}=20$ \\
\hline Coste fijo & Coste fijo \\
$F C_{i}=832$ & $F C_{i}=700$ \\
\hline
\end{tabular}

En cada una de las rondas $t(\mathrm{t}=1, \ldots, 15)$ del juego, los jugadores $i$ y $j(i, j=I$, $E$ con $i \neq j$ ), eligen simultáneamente si invertir en marketing/publicidad y fijan un precio para el producto que desean vender. Una demanda simulada por ordenador asocia una parte del mercado a los precios simultáneamente elegidos por los jugadores. Los pares precio-cantidad resultantes y los pagos son revelados ex post a los participantes.

Para el cálculo de los pagos introducimos un efecto inercia que vincula los pagos en cada ronda con las elecciones realizadas en rondas anteriores del juego ${ }^{4}$. Entonces, para cada jugador, la cantidad vendida depende no sólo de los precios actuales

\footnotetext{
${ }^{4}$ Mientras que hay unos pocos modelos teóricos de oligopolio que suponen demanda con inercia (veáse PHILIPS y RICHARD, 1989), este supuesto es bastante común en la literatura experimental: HOGGART (1959), SELTEN (1965), KESER (1992, 1993, 2000). Nuestro experimento difiere en que supone, al mismo tiempo, demanda con inercia y condiciones iniciales asimétricas.
} 
sino también de los precios anunciados en el pasado, y de la decisión de invertir en publicidad. En particular, esta última elección tiene el efecto de aumentar el impacto de la inercia en la demanda. Analíticamente:

$$
\begin{array}{lll}
q_{i, t}=\min \left[Q_{i, t}-\alpha\left(Q_{j, t}-\beta\right) s_{i, t-1}\right] & \text { si } & p_{i, t}>p_{j, t} \\
q_{i, t}=q_{i, t-1}+\gamma & \text { si } & p_{i, t}=p_{j, t}
\end{array}
$$

donde $Q_{i, t}$ es la cantidad que corresponde a $p_{i, t}, Q_{j, t}$ es la demanda total (es decir, la cantidad correspondiente al menor precio sobre la función de demanda) y $s_{i, t-1}$ es la cuota de mercado del jugador $i$ en la etapa previa del juego:

$$
s_{i, t-1}=\frac{q_{i, t-1}}{Q_{T, t-1}} \quad ; i, j=I, E
$$

Más concretamente, los parámetros de inercia $a, b, g$ funcionan de acuerdo a las siguientes expresiones:

$$
\text { Si } p_{i, t}>p_{j, t} \text { : }
$$

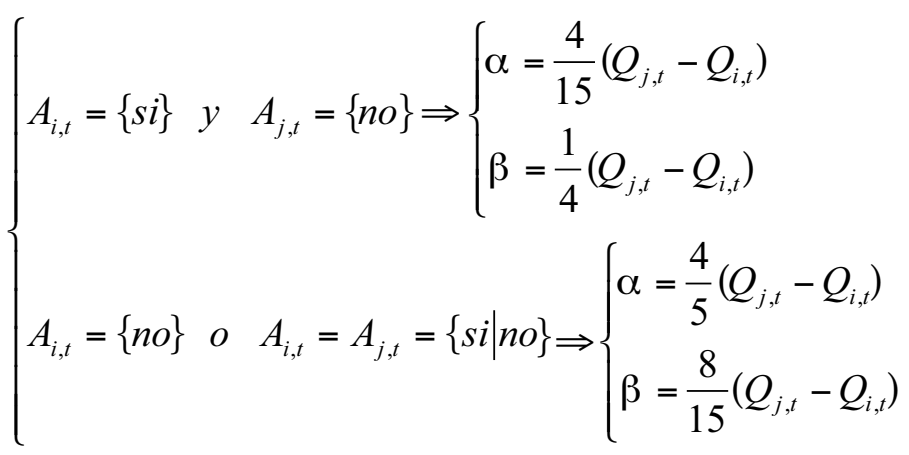

Si $p_{i, t}=p_{j, t}$ :

$$
\left\{\begin{array}{l}
A_{i, t}=A_{j, t}=\{s i \mid n o\} \Rightarrow \gamma=\frac{1}{2}\left|Q_{t}-Q_{t-1}\right| \\
A_{i, t}=\{s i\} \text { y } A_{j, t}=\{n o\} \Rightarrow\left\{\begin{array}{l}
\gamma=\frac{2}{3}\left|Q_{t}-Q_{t-1}\right| \text { if }\left(Q_{t}-Q_{t-1}\right)>0 \\
\gamma=\frac{1}{3}\left|Q_{t}-Q_{t-1}\right| \text { if } \quad\left(Q_{t}-Q_{t-1}\right)<0
\end{array}\right.
\end{array}\right.
$$

donde $A_{i, t}, A_{j, t}(i, j=I, E)$ representan el desembolso en publicidad (coste fijo), y $Q_{t}$ la cantidad sobre la curva de demanda en el caso de precios idénticos. Por lo tanto, para el caso $p_{i, t}>p_{j, t}$ el jugador $i$ pierde una cantidad que es proporcional bien a la diferencia de precios $\left(p_{i, t}-p_{j, t}\right)$ entre las dos empresas rivales, bien a su anterior cuota de mercado, con los parámetros $a$ y $b$ variando de acuerdo a la diferencia de precios. 
Además, suponemos que la publicidad tiene un efecto positivo en la participación de mercado siempre que la empresa ponga un precio más alto que el de su rival. Nótese que en el caso de precios idénticos, la publicidad conlleva un reparto de la demanda que es independiente de las cuotas de mercado previas. Por ello, sería irracional para el jugador E seguir la misma política de precios que la empresa I, ya que en cualquier caso sólo puede ganar, como máximo, 2/3 del incremento en la demanda.

Comenzamos con la identificación de una condición inicial para cada uno de los esquemas de demanda. Esta condición es la solución de equilibrio en el monopolio obtenida de la maximización de beneficios condicionada a la función de demanda considerada (Tabla 2). La idea subyacente a esta representación es que la firma I operaba como monopolista hasta el momento de la entrada de la firma $\mathrm{E}$.

La condición inicial se reemplaza en la primera ronda del experimento, de manera que la firma E puede entrar en el mercado. Como argumentaremos más adelante, en términos de las características de la industria, tal supuesto teórico tiene una significativa contrapartida empírica en los mercados en los que bien surge competencia tras expirar una patente, bien se liberaliza un monopolio natural.

Una vez que el mercado está abierto a la competencia, las empresas I y E operan al mismo coste unitario $c_{i}=20^{5}$. Las funciones de demanda, sin embargo, presentan un cierto efecto «inercia» exógeno ${ }^{6}$ que genera resultados de mercado asimétricos para los jugadores, es decir, diferentes cantidades y participaciones de mercado. Por lo tanto, estrategias de precios idénticas producen diferentes resultados para cada uno de los jugadores, dependiendo de la anterior posición del jugador en el mercado. Además, el efecto inercia puede verse reforzado/mitigado a través del «efecto marketing» si los jugadores deciden incurrir en costes fijos endógenos de markeging.

El tamaño de los costes fijos ha sido elegido de manera que se mantenga la misma solución teórica de Cournot-Nash para el correspondiente juego perfectamente simétrico de una jugada, en el que no son operativos ni el efecto inercia ni el efecto marketing. Los resultados numéricos se resumen en la Tabla 2.

\footnotetext{
${ }^{5}$ Este supuesto es equivalente a asumir, en el lenguaje de la literatura de organización industrial, la ausencia de costes de aprendizaje para el entrante o, en el lenguaje de la literatura de crecimiento, un completo derrame tecnológico.

${ }^{6}$ Ver expresiones (4) y (5).
} 
TABLA 2

SOLUCIONES PARA LOS CORRESPONDIENTES JUEGOS PERFECTAMENTE SIMÉTRICOS DE UNA JUGADA

Regulación $C$ sin costes de marketing

\begin{tabular}{|l|c|c|c|}
\hline & Monopolio & Cournot & Bertrand \\
\hline Precio & 70 & 53,4 & 20 \\
\hline Cantidad & 62,5 & $41,6 \times 2=83,2$ & $62,5 \times 2=125$ \\
\hline Beneficio & 3.125 & $1.389 \times 2=2.778$ & 0 \\
\hline Exc. consumidor & 0 & + & ++ \\
\hline
\end{tabular}

Regulación D sin costes de marketing

\begin{tabular}{|l|c|c|c|}
\hline & Monopolio & Cournot & Bertrand \\
\hline Precio & 85 & 63,5 & 20 \\
\hline Cantidad & 52 & $34,6 \times 2=69,2$ & $52 \times 2=104$ \\
\hline Beneficio & 3.380 & $1.505 \times 2=3.010$ & 0 \\
\hline Exc. consumidor & 0 & + & ++ \\
\hline
\end{tabular}

Regulación C con costes de marketing

\begin{tabular}{|l|c|c|c|}
\hline & Monopolio & Cournot & Bertrand \\
\hline Precio & 70 & 53,4 & 33,3 \\
\hline Cantidad & 62,5 & $41,6 \times 2=83,2$ & $62.5 \times 2=125$ \\
\hline Beneficio & 2.293 & $557 \times 2=1.114$ & 0 \\
\hline Exc. consumidor & 0 & + & ++ \\
\hline
\end{tabular}

Regulación D con costes de marketing

\begin{tabular}{|l|c|c|c|}
\hline & Monopolio & Cournot & Bertrand \\
\hline Precio & 85 & 63,5 & 33,46 \\
\hline Cantidad & 52 & $34,6 \times 2=69,2$ & $52 \times 2=104$ \\
\hline Beneficio & 2.425 & $805 \times 2=1.610$ & 0 \\
\hline Exc. consumidor & 0 & + & ++ \\
\hline
\end{tabular}




\section{Marco teórico}

Las usuales soluciones reducidas a modelos de duopolio de una jugada, a pesar de ser útiles a la hora de diseñar el experimento, no pueden ser consideradas como las soluciones teóricas de nuestro problema. En primer lugar, las herramientas habituales utilizadas para obtenerlas no se pueden aplicar en una situación en la que los jugadores realizan, de forma simultánea, elecciones sobre dos conjuntos de variables. En segundo lugar, nuestro experimento se ejecuta bajo la condición de información imperfecta. Los jugadores no saben ni la forma ni el tamaño de la función de demanda de mercado, la única información disponible que tienen es el precio inicial de equilibrio en monopolio. Tampoco saben nada acerca de los parámetros que describen la inercia y los efectos de marketing, a pesar de saber que estos efectos existen. Además, los jugadores desconocen que sus costes unitarios son idénticos. Por último, las soluciones tradicionales a modelos de una sola jugada no se aplican a las elecciones repetidas un número finito de veces, y tampoco las soluciones a los modelos secuenciales se aplican a una secuencia de elecciones simultáneas. Las técnicas habituales de equilibrio no se ajustan a modelos finitamente repetidos con señalización simultánea en dos dimensiones.

Sin embargo, al mirar la dinámica del juego uno puede al menos describir una estrategia óptima como el conjunto de movimientos secuenciales que conduce al pago acumulado más alto. Esto supondría para el entrante una estrategia que incrementa rápidamente su cuota de mercado de tal manera que compensa el mecanismo de inercia, para luego fijar precios de acuerdo a como hace la empresa establecida. A su vez, la mejor estrategia para la empresa establecida sería bien fijar precios altos e invertir en marketing/publicidad, o fijar precios ligeramente por encima de los del entrante sin incurrir en ningún coste de marketing/publicidad.

Distinguimos entre los siguientes subgrupos de estrategias: (1) estrategias que excluyen la entrada; (2) maximización del liderazgo en el mercado post-monopolio; (3) maximización de los beneficios post-monopolio. El primer subgrupo corresponde a estrategias de fijación de precios límite elegidas por la empresa establecida con el fin de prevenir la entrada en el mercado. El segundo y tercer subgrupos se refieren, respectivamente, al objetivo de la empresa establecida en cuanto a mantener la posición líder en el mercado el máximo tiempo posible, y a la maximización del beneficio global sin tener en cuenta la duración del liderazgo.

En términos de variables estratégicas disponibles para los jugadores, esto implica que la empresa establecida tiene dos variables para señalizar su comportamiento (precio y publicidad), mientras que la señalización por parte del entrante se limita a la política de precios elegida, y esto es cierto al menos para la etapa inicial del juego, en la que la combinación de baja dotación y cuota pequeña de mercado hacen difícil que el entrante pueda permitirse gastar en publicidad. Por lo tanto, la existencia de demanda con inercia, mientras supone un incentivo para una estrategia de señalización mixta por parte de la empresa establecida, limita el comportamiento del entrante al caso de señalización unidimensional. Además, las estrategias del entran- 
te son un subconjunto dado de estrategias de la empresa establecida, como veremos claramente más adelante.

En general, existen dos criterios de selección del equilibrio que se aplican al juego de múltiples etapas que tratamos aquí: procedimientos de estabilidad evolutiva con racionalidad limitada, y los procedimientos teóricos clásicos para los refinamientos del equilibrio de Nash. Trataremos estos dos criterios de forma separada.

\subsection{Estabilidad evolutiva}

Bajo el enfoque evolutivo, el equilibrio se define como la estabilidad de las elecciones a lo largo del tiempo, es decir, una situación en la cual el jugador juega siempre la misma estrategia contra su oponente. Buscamos una estrategia estable por parte de la empresa establecida distinguiendo entre dos tipos de comportamiento:

a) La empresa establecida adopta una estrategia «fuerte» contra la cual no existe una mejor respuesta por parte del entrante (Maynard Smith, 1974)

b) La empresa establecida adopta estrategias «débiles» a fin de (1) neutralizar al entrante (Maynard Smith, 1982) de manera que éste nunca gane más que la empresa establecida (equilibrio no simétrico), o bien (2) luchar contra la estrategia de óptima post-entrada del entrante (Swinkels, 1992) de manera que éste nunca gane el pago máximo post-entrada (no leapfrogging).

Donde b.1 y b.2 se refieren, respectivamente, al objetivo, por parte la empresa establecida, de mantener su posición de líder en el mercado y de maximizar el beneficio global sin importar la duración del liderazgo.

Consideremos ahora el «prototipo» de una matriz de pagos para la primera ronda del juego. Sin pérdida de generalidad, suponemos que los jugadores seleccionan precios en números discretos múltiples de diez en el rango [ $\mathrm{min}=$ coste de producción, $\max =$ precio de monopolio]. Esta situación está representada en la Figura 2. El conjunto de estrategias disponibles para el entrante es siempre un subconjunto de las estrategias disponibles para la firma establecida. Este subconjunto se ubica debajo de la diagonal noreste (fijación de precios simétrica) y viene representado por asteriscos $(*)$ en la Figura 2. Las celdas por encima de la diagonal noreste representan estrategias de bloqueo de entrada.

\footnotetext{
${ }^{7}$ Es decir, estrategias de bloqueo de entrada.
} 
FIGURA 2

MATRIZ DE PAGOS PROTOTIPO

\begin{tabular}{|l|c|c|c|c|c|c|c|}
\hline \multicolumn{1}{l}{$\mathrm{E}$} & $\mathrm{P}_{\mathrm{j}}=\mathbf{2 0}$ & $\mathrm{P}_{\mathrm{j}}=\mathbf{3 0}$ & $\mathrm{P}_{\mathrm{j}}=\mathbf{4 0}$ & $\mathrm{P}_{\mathrm{j}}=50$ & $\mathrm{P}_{\mathrm{j}}=60$ & $\mathrm{P}_{\mathrm{j}}=70$ & $\mathrm{P}_{\mathrm{j}}=\mathbf{8 0}$ \\
\hline $\mathrm{P}_{\mathrm{i}}=\mathbf{2 0}$ & & & & & & & \\
\hline $\mathrm{P}_{\mathrm{i}}=\mathbf{3 0}$ & ${ }^{*}$ & & & & & & \\
\hline $\mathrm{P}_{\mathrm{i}}=\mathbf{4 0}$ & ${ }^{*}$ & ${ }^{*}$ & & & & & \\
\hline $\mathrm{P}_{\mathrm{i}}=50$ & ${ }^{*}$ & ${ }^{*}$ & ${ }^{*}$ & & & & \\
\hline $\mathrm{P}_{\mathrm{i}}=60$ & ${ }^{*}$ & ${ }^{*}$ & ${ }^{*}$ & ${ }^{*}$ & & & \\
\hline $\mathrm{P}_{\mathrm{i}}=\mathbf{7 0}$ & ${ }^{*}$ & ${ }^{*}$ & ${ }^{*}$ & ${ }^{*}$ & ${ }^{*}$ & & \\
\hline $\mathrm{P}_{\mathrm{i}}=80$ & ${ }^{*}$ & ${ }^{*}$ & ${ }^{*}$ & ${ }^{*}$ & ${ }^{*}$ & ${ }^{*}$ & \\
\hline
\end{tabular}

Dada esta representación simplificada, en las Tablas 3, 4, 5 y 6 presentamos, para cada escenario, la matriz de pagos para la primera ronda del juego multi-etapas. Las cantidades correspondientes a las mejores estrategias de las primeras rondas se reportan en las matrices más pequeñas por debajo.

TABLA 3

ESTRATEGIAS DE EQUILIBRIO EN LA PRIMERA RONDA BAJO REGULACIÓN D Y SIN DESEMBOLSO EN PUBLICIDAD

\begin{tabular}{|c|c|c|c|c|c|c|c|}
\hline E & $P_{j}=20$ & $P_{j}=30$ & $P_{j}=40$ & $P_{j}=50$ & $P_{j}=60$ & $P_{j}=70$ & $P_{j}=80$ \\
\hline$P_{i}=20$ & 0 & 0 & 0 & 0 & 0 & 0 & 0 \\
\hline$P_{i}=30$ & 896 & 740 & 960 & 960 & 960 & 960 & 960 \\
\hline$P_{i}=40$ & 1.504 & ${ }_{144}^{1.632}$ & $\begin{array}{l}1.400 \\
360\end{array}$ & 1.760 & 1.7600 & $\begin{array}{ll}1.760 & \\
& 0\end{array}$ & 1.760 \\
\hline$P_{i}=50$ & $\begin{array}{ll}1.824 & \\
& 0\end{array}$ & $\begin{array}{l}2.016 \\
288\end{array}$ & $\begin{array}{r}2.208 \\
288\end{array}$ & $\begin{array}{r}1.980 \\
420\end{array}$ & $\begin{array}{r}2.400 \\
0\end{array}$ & $\begin{array}{l}2.400 \\
0\end{array}$ & 2.400 \\
\hline$P_{i}=60$ & 1.8560 & $\begin{array}{r}2.112 \\
432\end{array}$ & $\begin{array}{r}1.888 \\
816\end{array}$ & $\begin{array}{r}2.624 \\
432\end{array}$ & $\begin{array}{r}2.480 \\
400\end{array}$ & 2.8800 & 2.880 \\
\hline$P_{i}=70$ & 1.600 & $\begin{array}{l}1.920 \\
576\end{array}$ & $\begin{array}{l}2.540 \\
744\end{array}$ & $\begin{array}{r}2.560 \\
864\end{array}$ & $\begin{array}{l}2.880 \\
576\end{array}$ & $\begin{array}{l}2.900 \\
300\end{array}$ & 3.840 \\
\hline$P_{i}=80$ & $\begin{array}{l}1.056 \\
0\end{array}$ & $\begin{array}{r}1.440 \\
720 \\
\end{array}$ & $\begin{array}{r}1.824 \\
1152 \\
\end{array}$ & $\begin{array}{l}2.190 \\
1.305 \\
\end{array}$ & $\begin{array}{c}2.592 \\
1.152 \\
\end{array}$ & $\begin{array}{r}2.976 \\
720 \\
\end{array}$ & $\begin{array}{r}3.240 \\
120 \\
\end{array}$ \\
\hline
\end{tabular}

\begin{tabular}{|c|c|c|c|}
\hline E & $q_{j}\left(P_{j}=30\right)$ & $q_{j}\left(P_{j}=40\right)$ & $q_{j}\left(P_{j}=50\right)$ \\
\hline$q_{i}\left(P_{i}=60\right)$ & $\begin{array}{r}52,8(55 \%) \\
43,2(45 \%)\end{array}$ & $\begin{array}{r}47,2(54 \%) \\
40,8(46 \%)\end{array}$ & $\begin{array}{r}65,6(82 \%) \\
14,4(18 \%)\end{array}$ \\
\hline$q_{i}\left(P_{i}=70\right)$ & ${ }_{58,4(40 \%)}$ & ${ }_{30,8(58 \%)} 37,2(42 \%)$ & $\begin{array}{r}51,2(64 \%) \\
28,8(36 \%)\end{array}$ \\
\hline$q_{i}\left(P_{i}=80\right)$ & $24(25 \%) \quad 57,6(65 \%)$ & $30,4(35 \%){ }_{36,5(46 \%)}$ & $72(75 \%) \quad 43,5(54 \%)$ \\
\hline
\end{tabular}


TABLA 4

ESTRATEGIAS DE EQUILIBRIO EN LA PRIMERA RONDA BAJO REGULACIÓN D Y CON DESEMBOLSO EN PUBLICIDAD

\begin{tabular}{|c|c|c|c|c|c|c|c|}
\hline$E$ & $P_{j}=20$ & $P_{j}=30$ & $P_{j}=40$ & $P_{j}=50$ & $P_{j}=60$ & $P_{j}=70$ & $P_{j}=80$ \\
\hline$P_{i}=20$ & 0 & 0 & 0 & 0 & 0 & 0 & 0 \\
\hline$P_{i}=30$ & 239,20 & 113147 & 260 & 260 & 260 & 260 & 260 \\
\hline$P_{i}=40$ & $\begin{array}{l}976,8 \\
0\end{array}$ & $\begin{array}{r}1.018,4 \\
100,8\end{array}$ & 820240 & $\begin{array}{l}1.060 \\
0\end{array}$ & $\begin{array}{l}1.060 \\
0\end{array}$ & $\begin{array}{l}1.060 \\
0\end{array}$ & 1.060 \\
\hline$P_{i}=50$ & $\begin{array}{r}1.512,8 \\
0\end{array}$ & $\begin{array}{r}1.575,2 \\
201,6\end{array}$ & $\begin{array}{r}1.637,6 \\
201,6\end{array}$ & $\begin{array}{r}1.420 \\
282\end{array}$ & $\begin{array}{ll}1.700 & \\
& 0\end{array}$ & $\begin{array}{l}1.700 \\
0\end{array}$ & $\begin{array}{l}1.700 \\
0\end{array}$ \\
\hline$P_{i}=60$ & $\begin{array}{r}1.847,2 \\
0\end{array}$ & $\begin{array}{r}1.930,4 \\
302,4\end{array}$ & $\begin{array}{r}2.013,6 \\
403,2\end{array}$ & $\begin{array}{r}2.096,8 \\
302,4\end{array}$ & $\begin{array}{r}1.913,3 \\
268\end{array}$ & $\begin{array}{l}2.180 \\
0\end{array}$ & $\begin{array}{l}2.180 \\
0\end{array}$ \\
\hline$P_{i}=70$ & $\begin{array}{l}1.980 \\
0\end{array}$ & $\begin{array}{l}2.084 \\
403,2\end{array}$ & $\begin{array}{l}2.188 \\
\underline{604,8}\end{array}$ & $\frac{2.292}{\underline{604,8}}$ & $\begin{array}{l}2.396 \\
403,2\end{array}$ & $\begin{array}{r}2.300 \\
200\end{array}$ & 2.500 \\
\hline$P_{i}=80$ & $\begin{array}{r}61.911,2 \\
0\end{array}$ & $\begin{array}{r}2.036 \\
504\end{array}$ & $\begin{array}{l}\text { 2. } 160,8 \\
806,4\end{array}$ & $\begin{array}{r}2.285,6 \\
907,2\end{array}$ & $\begin{array}{l}2.410,4 \\
806,4\end{array}$ & $\begin{array}{r}2.535,2 \\
504\end{array}$ & $\begin{array}{r}2.580,2 \\
66,5\end{array}$ \\
\hline
\end{tabular}

\begin{tabular}{|c|c|c|c|}
\hline E & $q_{j}\left(P_{j}=30\right)$ & $q_{j}\left(P_{j}=40\right)$ & $q_{j}\left(P_{j}=50\right)$ \\
\hline$\left(P_{i}=70\right)$ & $\begin{array}{l}55,68(58 \%) \\
40,32(42 \%)\end{array}$ & $\begin{array}{l}57,66(65 \%) \\
30,24(35 \%)\end{array}$ & $\begin{array}{l}59,84(75 \%) \\
20,16(25 \%)\end{array}$ \\
\hline$\left(P_{i}=80\right)$ & $\begin{array}{c}45,6(47.5 \%) \\
50,4(52.5 \%)\end{array}$ & $\begin{array}{l}47,68(54 \%) \\
40,32(46 \%)\end{array}$ & $\begin{array}{l}49,76(62 \%) \\
30,24(38 \%)\end{array}$ \\
\hline
\end{tabular}


TABLA 5

ESTRATEGIAS DE EQUILIBRIO EN LA PRIMERA RONDA BAJO REGULACIÓN C Y SIN DESEMBOLSO EN PUBLICIDAD

\begin{tabular}{|c|c|c|c|c|c|c|}
\hline$E$ & $P_{j}=20$ & $P_{j}=30$ & $P_{j}=40$ & $P_{j}=50$ & $P_{j}=60$ & $P_{j}=70$ \\
\hline$P_{i}=20$ & 0 & 0 & 0 & 0 & 0 & 0 \\
\hline$P_{i}=30$ & 1.0250 & ${ }^{875} 250$ & 1.1250 & 1.1250 & 1.1250 & 1.125 \\
\hline$P_{i}=40$ & 1.600 & $\begin{array}{l}1.800 \\
225\end{array}$ & $\begin{array}{r}1.625 \\
375\end{array}$ & 2.000 & 2.000 & 2.000 \\
\hline$P_{i}=50$ & $\begin{array}{ll}1.725 & \\
& 0\end{array}$ & $\frac{2.025}{450}$ & $\frac{2.325}{450}$ & $\begin{array}{r}2.250 \\
375\end{array}$ & 2.6250 & 2.625 \\
\hline$P_{i}=60$ & 1.400 & $\begin{array}{l}1.800 \\
675\end{array}$ & $\begin{array}{r}2.200 \\
900\end{array}$ & $\begin{array}{r}2.600 \\
675\end{array}$ & $\begin{array}{r}2.750 \\
250\end{array}$ & 3.000 \\
\hline$P_{i}=70$ & 625 & $\begin{array}{c}1.125900 \\
900\end{array}$ & $\begin{array}{l}1.625 \\
1350\end{array}$ & $\begin{array}{l}2.125 \\
600\end{array}$ & $\begin{array}{l}2.625 \\
900\end{array}$ & 3.125 \\
\hline
\end{tabular}

\begin{tabular}{|c|c|c|}
\hline $\mathrm{E}$ & $q_{j}\left(P_{j}=30\right)$ & $q_{j}\left(P_{j}=40\right)$ \\
\hline$q_{i}\left(P_{i}=50\right)$ & $67,5(60 \%) \quad 45(40 \%)$ & $\begin{array}{l}77,5(77,5 \%) \\
22,5(22,5 \%)\end{array}$ \\
\hline$q_{i}\left(P_{i}=60\right)$ & $45(40 \%) \quad 67,5(60 \%)$ & $55(55 \%)$ \\
\hline
\end{tabular}

TABLA 6

ESTRATEGIAS DE EQUILIBRIO EN LA PRIMERA RONDA BAJO REGULACIÓN C Y CON DESEMBOLSO EN PUBLICIDAD

\begin{tabular}{|c|c|c|c|c|c|c|}
\hline I & $P_{j}=20$ & $P_{j}=30$ & $P_{j}=40$ & $P_{j}=50$ & $P_{j}=60$ & $P_{j}=70$ \\
\hline$P_{i}=20$ & $0 \quad 0$ & 0 & 0 & 0 & 0 & 0 \\
\hline$P_{i}=30$ & 260,50 & $\begin{array}{r}126,3 \\
166,7\end{array}$ & 293 & 293 & 293 & 293 \\
\hline$P_{i}=40$ & 1.0380 & $\begin{array}{l}1103 \\
157,5\end{array}$ & $\begin{array}{r}918 \\
250\end{array}$ & 1.1680 & 1.1680 & 1.168 \\
\hline$P_{i}=50$ & $\begin{array}{r}1.500,5 \\
0\end{array}$ & $\begin{array}{r}1.598 \\
315\end{array}$ & $\begin{array}{r}1.695,5 \\
315\end{array}$ & $\begin{array}{r}1.543,1 \\
249,9\end{array}$ & $\begin{array}{ll}1.793 & \\
& 0\end{array}$ & 1.793 \\
\hline$P_{i}=60$ & 1.6480 & $\begin{array}{l}1.778 \\
472,5\end{array}$ & $\frac{1.908}{\underline{630}}$ & $\begin{array}{l}2.038 \\
472,5\end{array}$ & $\begin{array}{r}2.001,3 \\
166,8\end{array}$ & 2.168 \\
\hline$P_{i}=70$ & $1.480,50$ & $\begin{array}{r}1.643 \\
630\end{array}$ & $\begin{array}{c}1.805,5 \\
945\end{array}$ & ${ }_{945}^{1.968}$ & $\begin{array}{r}2.130,5 \\
630\end{array}$ & 2.293 \\
\hline
\end{tabular}

\begin{tabular}{|c|c|c|c|}
\hline E & $q_{j}\left(P_{j}=30\right)$ & $q_{j}\left(P_{j}=40\right)$ & $q_{j}\left(P_{j}=50\right)$ \\
\hline$q_{i}\left(P_{i}=60\right)$ & $\begin{array}{l}65,25(58 \%) \\
47,25(42 \%)\end{array}$ & $\begin{array}{l}68,5(68,5 \%) \\
31,5(31,5 \%)\end{array}$ & $\begin{array}{r}71,75(82 \%) \\
15,75(18 \%)\end{array}$ \\
\hline$q_{i}\left(P_{i}=70\right)$ & $49,5(44 \%) \quad 63(56 \%)$ & $\begin{array}{c}52,75(52,7 \%) \\
47,25(47,3 \%)\end{array}$ & $56(64 \%) \quad 31,5(36 \%)$ \\
\hline
\end{tabular}


Por lo tanto, los jugadores racionales con información perfecta sobre el juego y comportamiento «miope» ${ }^{8}$ comenzarían por elegir una de las mejores estrategias de entre las presentadas arriba, alcanzando en algunos casos un equilibrio de Nash (celdas subrayadas en las Tablas 3 a 6 ).

Bajo estas condiciones particulares de estrategia de precios fijos, es posible calcular para cada escenario el número de rondas que les llevaría a los jugadores alcanzar cuotas iguales de mercado. Supongamos que la empresa entrante siempre juega la misma mejor estrategia para la primera ronda contra una estrategia constante por parte del entrante. El número de rondas necesarias para igualar las participaciones de mercado se presentan en la Tabla 7.

TABLA 7

RONDAS REQUERIDAS PARA IGUALAR

LAS PARTICIPACIONES DE MERCADO BAJO

UN COMPORTAMIENTO EVOLUTIVO

\begin{tabular}{|c|c|c|c|c|}
\hline \multicolumn{5}{|c|}{ Regulación C, No Marketing/Publicidad } \\
\hline & $\mathbf{E}$ & $\mathbf{P}_{\mathbf{j}}=\mathbf{3 0}$ & $\mathbf{P}_{\mathbf{j}}=\mathbf{4 0}$ & $\mathbf{P}_{\mathbf{j}}=\mathbf{5 0}$ \\
\hline $\mathbf{P}_{\mathbf{i}}=\mathbf{5 0}$ & 1 & 7 & \\
\hline $\mathbf{P}_{\mathbf{i}}=\mathbf{6 0}$ & 1 & 2 & 6 \\
\hline
\end{tabular}

\begin{tabular}{|c|c|c|c|c|}
\hline \multicolumn{5}{|c|}{ Regulación C, Marketing/Publicidad } \\
\hline & $\mathbf{E}$ & $\mathbf{P}_{\mathbf{j}}=\mathbf{3 0}$ & $\mathbf{P}_{\mathbf{j}}=\mathbf{4 0}$ & $\mathbf{P}_{\mathbf{j}}=\mathbf{5 0}$ \\
\hline $\mathbf{P}_{\mathbf{i}}=\mathbf{5 0}$ & 9 & Nunca & \\
\hline $\mathbf{P}_{\mathbf{i}}=\mathbf{6 0}$ & 4 & 7 & Nunca \\
\hline $\mathbf{P}_{\mathbf{i}}=\mathbf{7 0}$ & 1 & 2 & 6 \\
\hline
\end{tabular}

Regulación D, No Marketing/Publicidad

\begin{tabular}{|c|c|c|c|}
\hline E & $P_{j}=30$ & $P_{j}=40$ & $P_{j}=50$ \\
\hline$P_{i}=50$ & 5 & 12 & \\
\hline$P_{i}=60$ & 2 & 4 & 10 \\
\hline$P_{i}=70$ & 1 & 2 & 4 \\
\hline$P_{i}=80$ & 1 & 1 & 1 \\
\hline
\end{tabular}

Regulación D, Marketing/Publicidad

\begin{tabular}{|c|c|c|c|}
\hline I $\quad$ E & $P_{j}=30$ & $P_{j}=40$ & $P_{j}=50$ \\
\hline$P_{i}=50$ & 15 & Nunca & \\
\hline$P_{i}=60$ & 7 & 13 & Nunca \\
\hline$P_{i}=70$ & 3 & 5 & 11 \\
\hline$P_{i}=80$ & 1 & 2 & 5 \\
\hline
\end{tabular}

${ }^{8}$ Es decir, los jugadores maximizan los beneficios del presente periodo como si no hubiera un periodo siguiente (véase KESER, 1992). 
Las cifras en la Tabla 7 sugieren que tal solución al juego multi-etapas, mientras que consigue el bloqueo o retraso de la entrada, conlleva una gran cantidad de pérdidas en términos de beneficios acumulados. Al jugar constantemente una determinada estrategia, la empresa establecida está lejos de maximizar el beneficio global del juego. Buscamos, por lo tanto, un enfoque teórico alternativo. Nótese, sin embargo, que la partición del espacio de estrategias en la Figura 3 es independiente del método utilizado para definir la solución de equilibrio. En lo que sigue, mantenemos tal representación de la estrategia.

\subsection{Soluciones de Teoría de Juegos}

Dentro de este enfoque, nos referimos a los conceptos de perfección (Selten, 1975) y propiedad (Myerson, 1978) ${ }^{9}$. Para alcanzar pagos Pareto-superiores, el problema de coordinación -que es paralelo a la selección del equilibrio- se resuelve con el criterio de dominancia en riesgo utilizada para «normalizar» la matriz de pagos: cuanto más altos (bajos) los pagos de equilibrio, más pequeño (grande) el fallo de coordinación. Esto nos permite distinguir tres casos:

(a) Maximización del Pago para la Empresa Establecida (Empresa establecida «Fuerte»). Se identifican varias pautas de precios, dependiendo de que emerjan estrategias fuerte-débil. Pautas distintas se corresponden con diferentes estrategias de adaptación por parte del entrante.

(b) Maximización del Pago Total (Pareto Óptimo) para la empresa establecida y el entrante. Se selecciona la trayectoria de precios con riesgo recíproco mínimo (no hay efecto de dominancia en riesgo). Esta trayectoria nos da la solución simétrica.

(c) Maximización del Pago para el Empresa Entrante (Empresa entrante «Fuerte»). Se identifican varias pautas de precios posibles, dependiendo de las estrategias de reversión fuerte-débil. Diferentes pautas corresponden a diferentes estrategias de adaptación por parte de la empresa establecida.

El caso (a) se caracteriza por un equilibrio con separación que se asemeja a la solución de Stackelberg para el juego de líder-seguidor. Identificamos tales soluciones como una situación en la que los pagos acumulados de los jugadores difieren sustancialmente a lo largo del juego. Estas soluciones implican la aparición de una clara dominancia en riesgo por parte de la empresa establecida (Harsanyi and Selten, 1988).

\footnotetext{
${ }^{9}$ Estos conceptos difieren en la definición de robustez que adoptan. Bajo el concepto de perfección, la robustez se deriva de la baja probabilidad de errores. Bajo propiedad, la solidez se caracteriza por una baja probabilidad de errores costosos.
} 
El caso (b) es la retroalimentación de la solución de Nash para el juego simultáneo. El cálculo teórico de tal solución requiere un análisis numérico que está más allá de nuestros propósitos en este artículo ${ }^{10}$. En el Apéndice 3 presentamos un boceto del problema dinámico a resolver por medio de este análisis numérico. Este tipo de soluciones identifica una situación en la cual la dominancia en riesgo se compensa a través de la respuesta del oponente. Por lo tanto, el equilibrio converge a la solución simétrica.

Por último, llamamos a las soluciones del caso (c), «leapfrogging». Esto ocurre cuando el entrante, además de compensar por la cuota de mercado asimétrica, sobrepasa a la empresa establecida en términos de dominancia en riesgo.

\subsection{Implicaciones del criterio de equilibrio para el juego}

Desafortunadamente, los dos criterios de selección presentados anteriormente son débiles cuando se aplican al contexto que estamos analizando. Respecto al enfoque evolutivo, este tipo de razonamiento no se ajusta adecuadamente a nuestro problema de múltiples etapas porque no tiene en cuenta la reversibilidad de las elecciones a lo largo del tiempo, como sería el caso para las decisiones de marketing/ publicidad. De hecho, tal marco de análisis es más adecuado para tratar el caso de inversiones fijas como, por ejemplo, en capacidad adicional.

Por otra parte, el enfoque basado en la teoría de juegos también presenta algunos aspectos que están lejos de la situación modelizada en el experimento. La información imperfecta sobre el juego reduce drásticamente la posibilidad del jugador de seleccionar una trayectoria óptima y minimizar su distancia de ella.

Sin embargo, ambos intentos de solución son útiles para discutir nuestros resultados experimentales, ya que clarifican las estrategias utilizadas por los sujetos. En particular, ambos sugieren que las quince rondas del juego pueden ser reducidas a dos fases:

Fase 1: (Des)coordinación al comienzo del juego; ausencia/presencia de puntos focales. En esta fase, la cantidad vendida por la empresa establecida decrece mientras que las ventas del entrante se incrementan hasta alcanzar cuotas de mercado equilibradas. Esta fase está bien caracterizada por el enfoque evolutivo.

Fase 2: Esta fase del juego se capta dentro del enfoque basado en la teoría de juegos. Los jugadores alcanzan bien una solución de tipo Stackelberg, o juegan à la Cournot, o bien ocurre el leapfrogging.

\footnotetext{
${ }^{10}$ De hecho, los jugadores, en cualquier caso, no podrían implementar dicha solución debido a sus limitaciones de cálculo.
} 
En la fase uno, mientras que el entrante intenta incrementar su cuota de mercado, la empresa establecida puede adoptar bien una estrategia agresiva al reducir sus precios, bien puede mantener sus precios altos y utilizar los gastos en marketing/publicidad a fin de limitar su pérdida de cuota de mercado. Respecto a la fase dos, una vez que los jugadores comparten el mercado con cuotas más o menos iguales, éstos pueden alcanzar, bien un equilibrio de Stackelberg y mantener los precios diferenciados, bien fijar los mismos precios de manera que se alcanza un equilibrio de Cournot y, eventualmente, coordinar en precios más altos, o bien el entrante puede tratar de sobrepasar a la empresa establecida.

Estos resultados se presentan en la Figura 3. Las estrategias de entrada retardada presentan una asimetría persistente en las participaciones de mercado, a pesar de que dicha asimetría puede decaer con el tiempo.

FIGURA 3

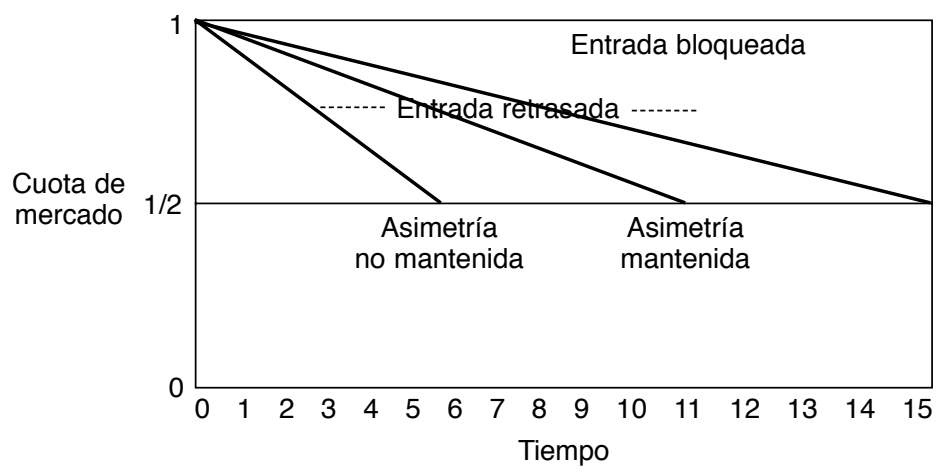

\section{El marco del experimento}

En cada etapa del juego los jugadores I y E compiten en la venta un producto homogéneo. La empresa establecida vende un producto con su marca, patentada en un periodo anterior, mientras el entrante vende un producto sin marca. Por lo tanto, los dos productos se consideran sustitutos perfectos por parte de los productores pero no por parte de los consumidores.

Modelizamos el mercado como un duopolio en precios con costes de producción simétricos y cuotas iniciales de mercado asimétricas. La empresa establecida tiene una dotación inicial de 10.000 procedente del periodo en el que tenía producción y distribución exclusivas, periodo que «idealmente» precede al juego de duopolio. En términos del efecto inercia, esto equivale a asignar, al principio del juego, todo el mercado al jugador I. En consecuencia, el entrante empieza con una cuota de mercado igual a cero y una dotación más baja, fijada en 3.000.

Existe un grupo de consumidores virtuales simulados por ordenador. Los efectos de la Regulación C y D son capturados por la elasticidad de la demanda. El experimento se realiza dos veces, cada vez con una función de demanda simulada diferente. 
El resultado de la transacción entre los jugadores I y E, es decir, los precios y cuotas de mercado resultantes, se muestran al final de cada ronda. Cada jugador tiene dos variables de decisión: el precio al cual desea vender su producto y el desembolso en marketing (publicidad). Dado que esta última variable se modela como un coste fijo, se representa como una elección cualitativa (SI o NO).

El experimento dura 15 rondas y se repite cuatro veces, de manera que cada sujeto juega ambos roles bajo las dos caracterizaciones institucionales. Por lo tanto, el experimento dura un total de 60 rondas. El propósito es detectar qué distribución de precios se obtendrá bajo cada caracterización de la demanda.

\section{Resultados experimentales}

El experimento se llevó a cabo en el Laboratorio de Economía de la Universidad de Siena. Duró aproximadamente tres horas y fue dividido en cuatro sesiones experimentales. Los sujetos fueron reclutados de los cursos de licenciatura en organización industrial y economía. Por lo tanto, todos ellos tenían conocimiento de teoría de juegos y algunos estaban familiarizados con experimentos de laboratorio.

Los 20 sujetos de cada sesión experimental fueron emparejados aleatoriamente por el ordenador, para formar 10 duopolios. El ordenador también asignó, al comienzo de cada sesión, un rol (empresa establecida o entrante) a cada participante. Esto se realizó de tal manera que, al cabo de las cuatro sesiones, todos los sujetos jugaran al menos una vez en ambos roles. Ninguno de los sujetos sabía quién era su oponente, ni los parámetros que describían la función de demanda a la cual se enfrentaban. Por lo tanto, no había posibilidad de aprendizaje de una sesión a otra.

Los sujetos tomaron sus decisiones en terminales de ordenador que estaban conectadas a través de una red local. Un programa instalado en un ordenador suplementario en la red convertía las decisiones de precio y publicidad, independientemente tomadas por los jugadores, en ventas y beneficios, y enviaba toda la información a los jugadores.

Antes de que comenzar el experimento se explicó el juego ${ }^{11}$ a los sujetos y se jugaron un par de rondas piloto para que se familiarizaran con el programa ${ }^{12}$. Además, se les sugirió que escribieran en una hoja especial la estrategia jugada así como el resultado de cada transacción, para facilitarles el razonamiento. Esta información también se podía consultar en la pantalla por medio de un menú.

Se pagó a los sujetos al final del experimento de acuerdo a su balance global durante el juego. Se aplicó un tipo de cambio al beneficio total de los jugadores para simplificar los pagos. Los sujetos también recibieron una cantidad fija por presentarse.

\footnotetext{
${ }^{11}$ Las instrucciones dadas a los participantes están disponibles bajo petición a los autores.

12 Utilizamos el programa z-Tree (FISCHBACHER, 2007) provisto por el Instituto de Investigación Empírica en Economía de la Universidad de Zurich.
} 
Ya comentamos en la sección 3 la imposibilidad de encontrar una solución basada en la teoría de juegos para la situación modelizada en el experimento. Allí también hipotetizamos sobre algunas posibles estrategias adoptadas por los jugadores que heurísticamente constituyen las mejores respuestas para la situación modelada en el juego. Estas soluciones parecen describir bastante bien algunas de las características del comportamiento observado en los participantes del experimento.

De hecho, el comportamiento de los sujetos en cada uno de los duopolios revela cuatro tipos de mercado:

- Establecida Fuerte - Entrante Débil: Las empresas alcanzan un equilibrio cercano a la solución de Stackelberg.

- Establecida Débil - Entrante Fuerte: El entrante sobrepasa a la empresa establecida (leapfrogging). La establecida adopta una estrategia de precios altos y no invierte en marketing. La E pone precios bajos hasta que $q_{E}$ es significativamente más grande que $q_{I}$ y luego aumenta el precio.

- Establecida Fuerte - Entrante Fuerte: Equilibrio cercano a la solución teórica de Cournot. Se produce maximización relativa del beneficio conjunto y mínima distancia entre los pagos.

- Establecida Débil - Entrante Débil: Las empresas alcanzan un equilibrio similar a la solución evolutiva. La E nunca alcanza la masa crítica $\left(q_{E}<q_{I}\right.$ siempre) y la inversión de los roles no altera el resultado.

La Tabla 8 presenta los beneficios medios a largo plazo de todos los mercados duopolísticos de las cuatro sesiones experimentales. Las celdas subrayadas indican que el sujeto jugó el papel de empresa establecida.

Nuestros resultados indican que los sujetos $4 \& 14$ y $8 \& 16$ están dentro del primer o segundo tipo de mercado, según el rol jugado por los sujetos. En particular, los sujetos 4 y 8 revelan un comportamiento fuerte en el que sobrepasan a sus adversarios cuando juegan como entrantes, y llegan a la solución de Stackelberg cuando juegan como empresas establecidas. Sus oponentes, en cambio, los sujetos 14 y 18, son débiles, con la única excepción del sujeto 14 bajo el Tratamiento I - Regulación C, que consigue llevar el equilibrio hacia una solución à la Cournot. Nótese, sin embargo, que el sujeto 4 tiene un mejor rendimiento, en términos de beneficios acumulados, que el sujeto 8 . Esto se debe al hecho de que este sujeto hizo un uso masivo de la publicidad incluso cuando jugaba como entrante, por lo que incurría en altos costes, sin ninguna posibilidad de apalancamiento en el precio. El sujeto 7 también presenta una actitud de fuerte, a pesar de que su comportamiento se caracteriza por frecuentes errores inexplicables.

Los sujetos $1 \& 11,2 \& 12,3 \& 13$ y $5 \& 15$ corresponden al tercer tipo de mercado. Con el tiempo, se ponen de acuerdo en un precio, del que se desvían sólo ligeramente. Este precio no difiere significativamente de la solución de Cournot en el juego de una sola jugada. Nótese que el sujeto 1 parece ser más fuerte que el sujeto 11 , en cuanto a que éste le sobrepasa cuando se comporta como entrante. Los suje- 
tos $3 \& 13$ en el Tratamiento I - Regulación C también convergen a la solución de Stackelberg.

Es interesante ver que los sujetos $2 \& 12$ están entre los que mejor coordinan sus estrategias. A pesar de que los beneficios son más altos para la empresa establecida, debido a la situación asimétrica inicial, sus pagos acumulados reflejan la distancia mínima relativa y están entre las más altas alcanzadas. El sujeto 2, sin embargo, parece ser un poco más fuerte que el sujeto 12 ya que tiende a sobrepasar a su rival cuando juega como entrante.

TABLA 8

BENEFICIOS ACUMULADOS DE LOS DUOPOLIOS

Trat. I - R Trat. I - E Trat. II - E Trat. II - R

Trat. I - R Trat. I - E Trat. II - E Trat. II - R

Trat. I - R Trat. I - E Trat. II - E Trat. II - R

Trat. I - R Trat. I - E Trat. II - E Trat. II - R

Trat. I - R Trat. I - E Trat. II - E Trat. II - R
Sujetos 1\&11

\begin{tabular}{|r|r|r|}
\multicolumn{1}{c|}{ suj. 1} & \multicolumn{1}{c|}{ suj. 11} & \multicolumn{1}{c|}{ Dif. } \\
\hline 31511 & 21701 & $\mathbf{9 8 1 0}$ \\
\hline 23074 & 25388 & $\mathbf{- 2 3 1 4}$ \\
\hline$\underline{35105}$ & 18150 & $\mathbf{1 6 9 5 5}$ \\
\hline 22146 & $\underline{23346}$ & $\mathbf{- 1 2 0 0}$ \\
\hline $\mathbf{1 1 1 8 3 6}$ & $\mathbf{8 8 5 8 5}$ & $\mathbf{2 3 2 5 1}$ \\
\hline
\end{tabular}

Sujetos $3 \& 13$

\begin{tabular}{|r|r|r|}
\multicolumn{1}{c}{ suj.3 } & \multicolumn{1}{c}{ suj. 13} & \multicolumn{1}{c|}{ Dif. } \\
\hline 29774 & 14986 & $\mathbf{1 4 7 8 8}$ \\
\hline 14461 & $\underline{42351}$ & $\mathbf{- 2 7 8 9 0}$ \\
\hline$\underline{31704}$ & 24806 & $\mathbf{6 8 9 8}$ \\
\hline 15106 & $\underline{36205}$ & $\mathbf{- 2 1 0 9 9}$ \\
\hline $\mathbf{9 1 0 4 5}$ & $\mathbf{1 1 8 3 4 8}$ & $\mathbf{- 2 7 3 0 3}$ \\
\hline
\end{tabular}

Sujetos 5\&15

\begin{tabular}{|r|r|r|}
\multicolumn{1}{r|}{ suj. 5} & suj. 15 & Dif. \\
\hline 29855 & 7642 & $\mathbf{2 2 2 1 3}$ \\
\hline 9764 & $\underline{27571}$ & $\mathbf{- 1 7 8 0 7}$ \\
\hline 25944 & 7026 & $\mathbf{1 8 9 1 8}$ \\
\hline 18141 & $\underline{23582}$ & $-\mathbf{5 4 4 1}$ \\
\hline $\mathbf{8 3 7 0 4}$ & $\mathbf{6 5 8 2 1}$ & $\mathbf{1 7 8 8 3}$ \\
\hline
\end{tabular}

Sujetos $7 \& 17$

\begin{tabular}{|r|r|r|}
\multicolumn{1}{c}{ suj. 7} & \multicolumn{1}{c|}{ suj. 17} & \multicolumn{1}{c|}{ Dif. } \\
\hline$\underline{27508}$ & 11951 & $\mathbf{1 5 5 5 7}$ \\
\hline 8691 & $\underline{27379}$ & $-\mathbf{- 1 8 6 8 8}$ \\
\hline$\underline{19885}$ & 31971 & $-\mathbf{1 2 0 8 6}$ \\
\hline 4939 & $\underline{30632}$ & $\mathbf{- 2 5 6 9 3}$ \\
\hline $\mathbf{6 1 0 2 3}$ & $\mathbf{1 0 1 9 3 3}$ & $-\mathbf{- 4 0 9 1 0}$ \\
\hline
\end{tabular}

Sujetos $9 \& 19$

\begin{tabular}{|r|r|r|}
\multicolumn{1}{c|}{ suj. 9} & \multicolumn{1}{c|}{ suj. 19} & \multicolumn{1}{c|}{ Dif. } \\
\hline$\underline{33176}$ & 8662 & $\mathbf{2 4 5 1 4}$ \\
\hline 22719 & 19312 & $\mathbf{3 4 0 7}$ \\
\hline 24823 & 13395 & $\mathbf{1 1 4 2 8}$ \\
\hline 11721 & $\underline{34615}$ & $\mathbf{- 2 2 8 9 4}$ \\
\hline $\mathbf{9 2 4 3 9}$ & $\mathbf{7 5 9 8 4}$ & $\mathbf{1 6 4 5 5}$ \\
\hline
\end{tabular}

Sujetos $2 \& 12$

\begin{tabular}{|r|r|r|}
\multicolumn{1}{c}{ suj. 12} & \multicolumn{1}{c|}{ suj. 2} & \multicolumn{1}{c|}{ Dif. } \\
\hline$\underline{35875}$ & 19990 & $\mathbf{1 5 8 8 5}$ \\
\hline 20282 & $\underline{34144}$ & $\mathbf{- 1 3 8 6 2}$ \\
\hline$\underline{28768}$ & 23885 & $\mathbf{4 8 8 3}$ \\
\hline 18605 & $\underline{35560}$ & $\mathbf{- 1 6 9 5 5}$ \\
\hline $\mathbf{1 0 3 5 3 0}$ & $\mathbf{1 1 3 5 7 9}$ & $-\mathbf{- 1 0 0 4 9}$ \\
\hline
\end{tabular}

Sujetos 14\&4

\begin{tabular}{|r|r|r|}
\hline suj.14 & \multicolumn{1}{c}{ suj.4 } & \multicolumn{1}{c|}{ Dif. } \\
\hline$\underline{26787}$ & 29390 & -2603 \\
\hline 18259 & $\underline{30150}$ & -11891 \\
\hline$\underline{24977}$ & 28863 & -3886 \\
\hline 14453 & 38384 & $-\mathbf{2 3 9 3 1}$ \\
\hline $\mathbf{8 4 4 7 6}$ & $\mathbf{1 2 6 7 8 7}$ & -42311 \\
\hline
\end{tabular}

Sujetos 16\&6

\begin{tabular}{|r|r|r|}
\multicolumn{1}{r|}{ suj. 16} & suj. 6 & \multicolumn{1}{c|}{ Dif. } \\
\hline 18858 & 3503 & 15355 \\
\hline 2098 & $\underline{18880}$ & -16782 \\
\hline 19173 & 7769 & 11404 \\
\hline 4507 & $\underline{23827}$ & -19320 \\
\hline 44636 & 53979 & -9343 \\
\hline
\end{tabular}

\section{Sujetos $18 \& 8$}

\begin{tabular}{|r|r|r|}
\multicolumn{1}{c}{ suj. 18} & \multicolumn{1}{c}{ suj. 8} & \multicolumn{1}{c|}{ Dif. } \\
\hline$\underline{24222}$ & 23433 & $\mathbf{7 8 9}$ \\
\hline 15578 & $\underline{27587}$ & -12009 \\
\hline 16757 & 26833 & -10076 \\
\hline 5808 & $\underline{22056}$ & -16248 \\
\hline $\mathbf{6 2 3 6 5}$ & $\mathbf{9 9 9 0 9}$ & -37544 \\
\hline
\end{tabular}

Sujetos 20\&10

\begin{tabular}{|r|r|r|}
\multicolumn{1}{c}{ suj. 20} & \multicolumn{1}{c}{ suj. 10} & \multicolumn{1}{c|}{ Dif. } \\
\hline$\underline{26078}$ & 14385 & $\mathbf{1 1 6 9 3}$ \\
\hline 30285 & $\underline{15540}$ & $\mathbf{1 4 7 4 5}$ \\
\hline 32958 & 7638 & $\mathbf{2 5 3 2 0}$ \\
\hline 26917 & $\underline{25987}$ & $\mathbf{9 3 0}$ \\
\hline $\mathbf{1 1 6 2 3 8}$ & $\mathbf{6 3 5 5 0}$ & $\mathbf{5 2 6 8 8}$ \\
\hline
\end{tabular}


Finalmente, los sujetos $6 \& 16$ reflejan una solución evolutiva. A pesar de que el resultado global es una solución de Stackelberg, existe un bajo nivel de aspiración por parte de la empresa establecida, la cual comienza con un precio sustancialmente más bajo que la solución de Cournot-Nash, mientras que al mismo tiempo el entrante exhibe una conducta imitativa de adaptación a la política de precios de la empresa establecida. Sin embargo, el comportamiento de estos sujetos parece irracional, ya que coordinan de forma persistente sus estrategias hacia precios iguales al coste marginal, lo que implica que sus beneficios son bajos o incluso nulos.

Los tipos de mercado pueden identificarse también por el nivel de precios medios de mercado (ver Tabla 9). Aquí distinguimos mercados cooperativos que muestran poca variabilidad de precios, y precios más altos que los mercados débilmente cooperativos en los que hay mayor inestabilidad de precios. Los mercados $2 \& 12,3 \& 13$, $6 \& 16,7 \& 17$ y $9 \& 19$ caen dentro del primer grupo. En estos mercados, los diferenciales medios de precios entre las dos empresas rivales no exceden los 10 puntos y los precios tienden a ser los más altos. Los mercados $1 \& 11,14 \& 4,5 \& 15,18 \& 8$ y $20 \& 10$ son, en cambio, débilmente cooperativos. Muestran diferenciales de precios más grandes que los mercados cooperativos, y niveles de precios que son más bajos.

A nivel agregado se obtienen también resultados interesantes. El análisis de las cantidades medias revela que la elasticidad de la demanda no influye en los precios de equilibrio. Los precios medios convergen a una solución de tipo Cournot tanto bajo Regulación C (demanda elástica) como Regulación D (demanda rígida), a pesar de que en el último caso la convergecia en cantidades es más lenta.

TABLA 9

PRECIOS MEDIOS DE MERCADO

\begin{tabular}{|c|c|c|c|}
\hline & \multicolumn{3}{|c|}{ Tratamiento I - Regulación D } \\
\hline & $\mathbf{P i}$ & pj & pi - pj \\
\hline $1 \& 11$ & 70 & 36 & 34 \\
\hline $12 \& 2$ & 80 & 70 & 10 \\
\hline $3 \& 13$ & 50,5 & 45 & 5,5 \\
\hline $14 \& 4$ & 66 & 50 & 16 \\
\hline $5 \& 15$ & 46,5 & 61,5 & -15 \\
\hline $16 \& 6$ & 28 & 29 & -1 \\
\hline $7 \& 17$ & 43,5 & 32 & 11,5 \\
\hline $18 \& 8$ & 72 & 51,5 & 20,5 \\
\hline $9 \& 19$ & 54 & 50 & 4 \\
\hline $20 \& 10$ & 67 & 52 & 15 \\
\hline & Tratar & II - $\mathbf{R}$ & ación C \\
\hline & $\mathbf{P i}$ & pj & pi - pj \\
\hline $1 \& 11$ & 67,5 & 68 & $-0,5$ \\
\hline $12 \& 2$ & 66,5 & 55 & 11,5 \\
\hline $3 \& 13$ & 57 & 51,5 & 5,5 \\
\hline $14 \& 4$ & 62,5 & 47,5 & 15 \\
\hline $5 \& 15$ & 31,5 & 40 & $-8,5$ \\
\hline $16 \& 6$ & 27,5 & 29,5 & -2 \\
\hline $7 \& 17$ & 60,5 & 44 & 16,5 \\
\hline $18 \& 8$ & 52,5 & 33,5 & 19 \\
\hline $9 \& 19$ & 55 & 37 & 18 \\
\hline $20 \& 10$ & 39 & 39 & 0 \\
\hline
\end{tabular}

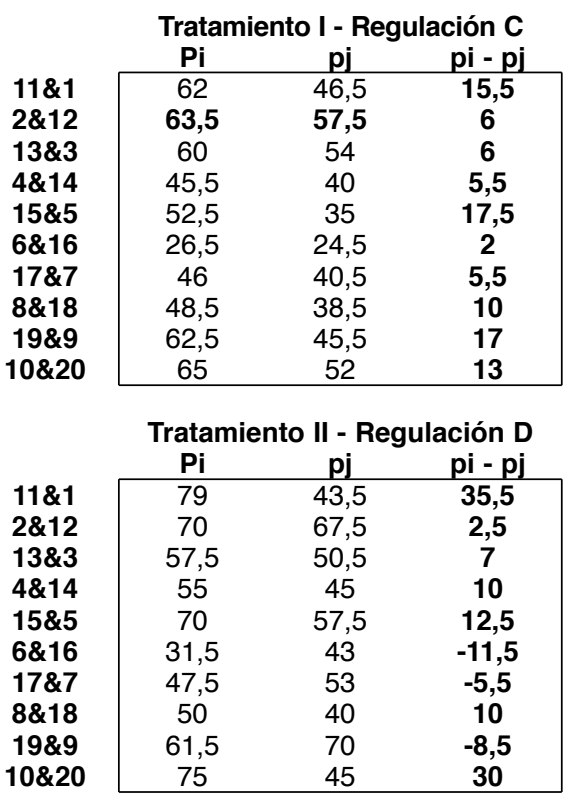


Finalmente, los resultados revelan una estructura de fases dentro del juego. Casi todas las estrategias reflejan una fase inicial que precede a la fase principal. La fase inicial fija el precio de entrada y sirve para señalizar la voluntad de cooperar. Esta fase dura hasta que el entrante alcanza a la empresa establecida en términos de cuota de mercado. La fase principal caracteriza el equilibrio de acuerdo a la actitud cooperativa de los jugadores.

\section{Conclusiones}

Nuestros resultados muestran que, bajo información imperfecta, un recorte secuencial de precios que lleve a la solución competitiva es difícil de obtener en un juego de múltiples etapas. Los jugadores raramente se coordinan hacia una solución de tipo Bertrand, pues tal comportamiento conduciría a beneficios bajos o nulos. Por el contrario, los beneficios positivos parecen ser una característica general de los modelos multi-etapa en los que el mercado se caracteriza por una empresa establecida que es dominante y una empresa periférica que entra al mercado.

Además, con inercia en la demanda, la empresa establecida alcanza beneficios mayores que los del entrante y tal ventaja de único jugador se suaviza muy lentamente, especialmente si la empresa establecida muestra una actitud fuerte. De los diez duopolios experimentales, siete reflejan esta característica. En estos mercados, los sujetos, bien se coordinan hacia una solución de Cournot, bien alcanzan un equilibrio de Stackelberg.

Sugerimos varias aplicaciones para este tipo de situaciones. Consideremos, por ejemplo, mercados que se abren a la competencia tras una situación de monopolio. Nuestros resultados muestran que la persistencia de la ventaja de único jugador, incluso tras la liberalización, dificulta la obtención de un mercado perfectamente disputable (contestable) sólo por permitir la entrada de otra empresa ${ }^{13}$. Los incentivos privados no son suficientes para crear una situación competitiva en el caso en que el poder de mercado está distribuido asimétricamente entre los competidores. Además, el poder de mercado decae lentamente incluso cuando la empresa establecida se enfrenta a un entrante fuerte.

Otra aplicación es la competencia que surge tras expirar una patente. Tras un periodo de producción y distribución exclusivas, nuestros resultados indican que la ventaja competitiva se mantiene más allá de la expiración legal. Hudson (2000) se refiere al caso de la industria farmacéutica. Encuentra que, cuanto mayor el mercado, mayor la probabilidad de que ocurra tanto la entrada del genérico como el subsiguiente impacto en las ventas de la empresa establecida. Nuestros resultados con-

\footnotetext{
${ }^{13}$ Recuérdese que esta es la condición más favorable en la cual la entrada de más de una empresa incrementaría la ventaja de único jugador que tiene la empresa establecida. Sobre estos temas, véase la discusión de Sherman y Willett sobre los efectos de la entrada múltiple en los modelos de oligopolio de Bain-Sylos (SHERMAN y WILLETT, 1967).
} 
firman sólo parcialmente esta evidencia. A pesar de que las empresas entrantes consigan igualar su cuota con la establecida, persiste una gran asimetría, en términos de beneficios obtenidos, a lo largo de todo el juego. Sólo tres de los diez duopolios investigados confirman este resultado.

En este sentido, los resultados de nuestro experimento ofrecen, de manera implícita, una idea sobre la vida efectiva de la patente. En referencia a la importancia de esta cuestión para la industria farmacéutica, observamos que la vida efectiva de una patente farmacéutica se reduce drásticamente por la longitud del proceso de desarrollo de la droga y, particularmente, en lo que se refiere a las pruebas clínicas. Nuestros resultados indican que esta situación es, al menos parcialmente, compensada por la explotación del poder de mercado tras expirar la patente. Incluso cuando se enfrentan a un entrante agresivo, las empresas establecidas tienen buenas oportunidades de mantener sus ventajas, especialmente en el contexto de una demanda de mercado segmentada. De hecho, dado que el ciclo vital de una droga no termina con la fecha de expiración de su patente, existe margen para continuar con el disfrute de los beneficios extraordinarios.

Los sujetos experimentales también nos muestran que un comportamiento de tipo Cournot es siempre preferible a una solución coordinada en términos de excedente del consumidor. Esto es así especialmente cuando la firma establecida utiliza racionalmente el nivel de publicidad. Recordemos que, en el contexto analizado, la publicidad, a pesar de ser modelizada como un coste fijo, no representa una elección irreversible a lo largo del juego. Sin embargo, la firma establecida puede hacer que esta opción sea irreversible gastando constantemente en publicidad hasta que el mercado se comparta, a partes iguales, con el entrante. Entonces, la empresa establecida puede mandar la señal de un comportamiento más suave que, si es correctamente detectada y aceptada por el entrante, conduce a una solución cooperativa con precios finales más altos que en la solución de Cournot estática. Nótese también que la mayoría de los duopolistas de tipo Cournot tienden a coordinarse, al final del juego, hacia precios más altos que los de la solución de Cournot estática. Esto confirma los resultados de Keser (2000), que muestran que, con costes simétricos, los duopolios presentan un significativo comportamiento cooperativo.

Sin embargo, el efecto de señalización de la publicidad no se determina unívocamente. La falta de publicidad por parte de la empresa establecida limita el equilibrio a una solución de bajo nivel que se asemeja a la competencia à la Bertrand. Por lo tanto, nuestras dos dimensiones de señalización implican que la señal del precio será siempre más fuerte que la de la publicidad, y que el uso a largo plazo de la señal, conduce irrevocablemente a una trampa de bajos beneficios tanto para la empresa establecida como para la entrante. 


\section{Bibliografía}

[1] ARROW, K.J., (1962). «Economic welfare and the allocation of resources for invention», en The rate and direction of inventive activity: economic and social factors, Conference No. 13, Universities-National Bureau of Economic Research, Princeton: Princeton University Press.

[2] BASS, F.M., EDGAR, A., PESSEMIER y D.R. LEHMANN (1972). «An experimental study of relationships between attitudes, brand, preference and choice», Behavioral Science, 17, 6, pp. 532-541.

[3] DeJONG, D.V., R. FORSYTHE, J. W. SCHATZBERG y W. C. UECKER (1996). «Collusion and product quality: A laboratory investigation», en Research in Experimental Economics, 6, pp. 1-51. Greenwich, Connecticut: JAI Press.

[4] DIXIT, A.K. (1980). «The role of investment in entry-deterrence», Economic Journal, 90, pp. 95-106.

[5] DORFMAN, R. y R.L. STEINER (1954). «Optimal advertising and optimal quality», American Economic Review, 44, pp. 826-836.

[6] FISCHBACHER, U. (2007). «z-Tree: Zurich toolbox for readymade economic experiments. Experimenter's manual», Experimental Economics, 10, 2, pp. 171-178.

[7] FISHER, K. (1988). Oligopolistiche marktprozesse: Einsatz verschiedener preis-mengen-strategien unter berücksichtinung von Nachtfrageträgheit, Heidelberg: PhysicaVerlag.

[8] FRIEDMAN, J. W. (1979). «On entry preventing behavior and limit price models of entry», en: Applied Game Theory, editado por S.J. Brams, A. Schotter y G. Schwodiauer, pp. 236-253, Wurzburg: Physica-Verlag.

[9] FRIEDMAN, J.W. (1983). Oligopoly theory, Cambridge: Cambridge University Press.

[10] FUDENBERG, D. y J. TIROLE (1984). «The fat-cat effect, the puppy-dog ploy, and the lean and hungry look», American Economic Review, 74, pp. 361-366.

[11] GILBERT, R.J. y D.M.G. NEWBURY (1982). «Preemptive patenting and the persistence of monopoly», American Economic Review, 72, pp. 514-26.

[12] HOGGART, A.C. (1959). «An experimental business game», Behavioral Science, 4, pp. 192-203.

[13] HUDSON, J. (2000). «Generic take-up in the pharmaceutical market following patent expiry. A multi-country study», International Review of Law and Economics, 20, pp. 205-221.

[14] HURWITZ, M.A. y R.E. CAVES (1988). «Persuasion or information? Promotion of the shares of brand name and generic pharmaceuticals», Journal of Law \& Economics, 31, 299-320.

[15] KESER, C. (1992). «Experimental duopoly markets with demand inertia -game playing experiments and the strategy method»(Lecture notes in economic and mathematical systems), vol. 391. Berlin:Springer.

[16] KESER, C. (1993). «Some results of experimental duopoly markets with demand inertia», Journal of Industrial Economics, 41, pp. 133-151.

[17] KESER, C. (2000). «Cooperation in symmetric duopolies with demand inertia», International Journal of Industrial Organization, 18, pp. 23-38.

[18] KREPS, D.M. y R. WILSON (1982). «Reputation and imperfect information», Journal of Economic Theory, 27, 253-279. 
[19] LIEBERMAN, M.V. y D.B. MONTGOMERY (1988). «First-mover advantages», Strategic Management Journal, 9, pp. 41-58.

[20] MILGROM, P.P. y J. ROBERTS (1982). «Limit pricing and entry under incomplete information: an equilibrium analysis», Econometrica, 50, pp. 443-460.

[21] MODIGLIANI, F. (1958). «New developments on the oligopoly front», Journal of Political Economy, 66, pp. 215-232.

[22] MUELLER, D.C. (1997). «First-mover advantages and path-dependence», International Journal of Industrial Organization, 15, 6, pp. 827-850.

[23] MYERSON, R.B. (1978). «Refinements of the Nash equilibrium concept», International Journal of Game Theory, 7, pp. 73-80.

[24] NERLOVE, M. y K.J. ARROW (1962). «Optimal advertising policy under dynamic conditions», Economica, 29, pp. 129-142.

[25] PEPALL, L., RICHARDS, D.J. y G. NORMAN (1999). Industrial organization: contemporary theory and practice, South-Western College Publishing.

[26] PLOTT, C.R. y L.L. WILDE (1982). «Professional diagnosis vs. self-diagnosis: an experimental examination of some special features of markets with uncertainty», en Research in Experimental Economics, 2, pp. 63-112. Greenwich, Connecticut: JAI Press.

[27] REINGANUM, J.F. (1983). «Uncertain innovation and the persistence of monopoly», American Economic Review, 73, pp. 741-748.

[28] ROSENTHAL, R.W. (1981). «Games of perfect information, predatory pricing and the chain-store paradox», Journal of Economic Theory, 25, pp. 92-100.

[29] SHERMAN, R. y T.D. WILLETT (1967). «Potential entrants discourage entry», Journal of Political Economy, 55, pp. 400-403.

[30] SCHMALENSEE, R. (1983). «Advertising and entry-deterrence: an exploratory model», Journal of Political Economy, 90, pp. 636-653.

[31] SELTEN, R. (1965). «Spieltheoretische behandlung eines oligopolmodells mit nachtfragetragheit», Zeitschrift fur die gesamte staatwissenschaft, 121, pp. 301-24 y 667-689.

[32] SPENCE, A.M. (1979). «Investment strategy and growth in a new market», Bell Journal of Economics, 10, pp. 1-19.

[33] SYLOS LABINI, P. (1962). Oligopoly and technical change, Cambridge, Mass., Harvard Un. Press. 